\title{
Article
}

\section{Particle Dispersion/Aggregation Model in a Non-Uniform Shear Flow}

\author{
Koji MASUdA", Hiroshi SuZuki ${ }^{* \dagger}$, Yoshiyuki KomodA*, and Ruri HIDEMA ${ }^{* *}$ \\ "Department of Chemical Science and Engineering, Kobe University \\ 1-1, Rokkodai-cho, Nada-ku, Kobe-shi, Hyogo 657-8501, Japan \\ ** Organization of Advanced Science and Technology, Kobe University \\ 1-1, Rokkodai-cho, Nada-ku, Kobe-shi, Hyogo 657-8501, Japan \\ (Received : December 13, 2012)
}

\begin{abstract}
A model estimating local particle dispersion/aggregation behaviors in a suspension has been developed for a nonuniform shear flow. In this model, the size distributions of particle clusters were calculated with taking a balance of Brownian and shear coagulations and shear breakup. A numerical simulation was performed for a two-dimensional backward-step flow as a non-uniform shear flow. A flow experiment using a micro-channel with a rib was also conducted for the verification of the present numerical analysis. The solid volume fraction of the suspension was set at 0.01 and the step height Reynolds number was kept constant at $1.5 \times 10^{-4}$ both for numerical and for experimental studies. The numerical results obtained in this study were rather reasonable compared with the experimental data. From this, it was found that the present numerical model is promising for estimating particle dispersion/aggregation in a non-uniform shear flow.
\end{abstract}

Key Words: Suspension / Dispersion/Aggregation / Polymer composite / Fine particle

\section{INTRODUCTION}

The suspension systems are frequently used for many industrial processes such as ceramics, pharmaceuticals, cosmetics and polymer composites. Among them, polymer composites and nanoparticle composites, which are made from solid-liquid suspensions with inorganic fine particles in molten polymers, have received a great deal of attention recently in order to add some functions to polymers. However, fine particles can easily aggregate due to the inter-particle interaction. This causes degradation of performances of transparence, stiffness, tension stability, etc. These phenomena appear to be the cause of the degradation of the products. Moreover, local heterogeneous size and shape distributions of aggregates (clusters) severely affect the products rates in the manufacturing process. Thus, it is of importance to prevent the local aggregation and to predict aggregation characteristics. The particle behaviors in solid-liquid suspensions are affected by several internal and external factors. Smoluchowski ${ }^{1)}$ investigated the basic particle contact model for solidliquid suspensions and slurries. Swift et al. ${ }^{2)}$ performed an experimental study about Brownian coagulation in suspensions. Higashitani et al. ${ }^{3-5)}$ worked out the coagulation model of fine particles to estimate time-dependence of total particle concentration in suspensions. Parker et al. ${ }^{6}$ revealed that the rupture and erosion are two major factors for the mechanism of particle cluster breakup in suspensions. Sonntag et $a l^{7-9)}$ investigated the relationship between the cluster size and the deformation rate. Usui ${ }^{10)}$ developed a model to predict the change rate of the cluster size and the bulk suspension viscosity considering the Brownian coagulation, the shear coagulation and the shear breakup of clusters. Some experimental studies ${ }^{11,12)}$ have supported the validation of the model. In the Usui's model, however, the cluster size was assumed to be uniform. In actual cases, cluster size widely distributes in suspensions. Hasegawa et al. ${ }^{13)}$ modified the Usui's model and developed a model to predict the cluster size distributions and the rheological characteristics of solidliquid suspensions. Hasegawa's model was applied to the experimental results ${ }^{14)}$ and was verified. ${ }^{15)}$ However, the model developed by Hasegawa et al. as well as Usui's model could be applied only to a simple shear flow having a uniform shear rate. An actual flow field is complex and the shear rate changes locally in some manufacturing processes.

In this study, a model to predict local dispersion/aggregation characteristics of particle clusters in solid-liquid suspensions in a flow with non-uniform shear distributions was developed. A two-dimensional numerical simulation was applied to a flow with a backward facing step. Experiments using a micro-channel with a rib mounted on one-side wall were also performed to verify the model.

$\dagger$ hero@kobe-u.ac.jp 


\section{METHOD}

\subsection{Numerical Methods}

In this study, a coupling model between the coagulation behavior analysis based on Usui's and Hasegawa's models and the two-dimensional flow calculation were applied to a backward-facing flow. Usui ${ }^{11)}$ developed a model to predict the behaviors of particle coagulations and break-ups in highconcentrated suspensions considering Brownian coagulation, shear coagulation and shear breakup of particles as shown in the following

$$
\frac{\mathrm{d} k}{\mathrm{~d} t}=\frac{4 \alpha_{\mathrm{b}} k_{\mathrm{b}} T N_{0}}{3 \eta_{0}}+\frac{4 \alpha_{\mathrm{s}} \phi k \dot{\gamma}}{\pi}-\frac{3 \pi d_{0}^{3} k}{4 F_{0} N_{\mathrm{b}}}\left(\frac{k}{1-\varepsilon}-1\right) \eta \dot{\gamma}^{2}
$$

Here $k[-]$ is the number of particles in a cluster, $t[\mathrm{~s}]$ is time, $k_{\mathrm{b}}\left[\mathrm{J} \cdot \mathrm{K}^{-1}\right]$ is Boltzmann constant, $T[\mathrm{~K}]$ is the temperature of fluids and $N_{0}\left[\mathrm{~m}^{-3}\right]$ is the total number of particles per unit volume. $\phi[-]$ is the apparent solid volume fraction, $\varepsilon$ [-] is the void fraction of particles in a cluster and $d_{0}[\mathrm{~m}]$ is the diameter of a primary particle. $\eta[\mathrm{Pa} \cdot \mathrm{s}]$ is the suspension viscosity, $\eta_{0}[\mathrm{~Pa} \cdot \mathrm{s}]$ is the solvent viscosity and $\dot{\gamma}\left[\mathrm{s}^{-1}\right]$ is the shear rate add to the suspensions. $\alpha_{\mathrm{b}}(=0.58)[-]$ and $\alpha_{\mathrm{s}}(=0.60)[-]$ are the kinetic constants for Brownian coagulation and for shear coagulation obtained by Higashitani. ${ }^{3,4)} F_{0}[\mathrm{~J}], N_{\mathrm{b}}[-]$ are the bonding energy between particles and the number of bonds to be broken when the cluster is divided into two same size clusters. $N_{\mathrm{b}}$ is estimated as follows

$$
N_{\mathrm{b}}=\frac{k d_{0}}{2 d_{\mathrm{k}}}
$$

Here, $d_{\mathrm{k}}[\mathrm{m}]$ is the diameter of cluster with $k$ particles.

$F_{0}$ is calculated from the experimental viscosity data in a steady state using a rheometer as reported in the previous studies. ${ }^{14)}$ Hasegawa et al. modified the model in order to predict time-dependent cluster size distributions as follows

$$
\begin{aligned}
\frac{\mathrm{d} n_{\mathrm{k}}}{\mathrm{d} t} & =\frac{1}{2} \alpha_{\mathrm{b}} \sum_{i=1}^{i=j=1} \frac{2 k_{\mathrm{b}} T}{3 \eta_{0}}\left(r_{\mathrm{i}}+r_{\mathrm{j}}\right)\left(\frac{1}{r_{\mathrm{i}}}+\frac{1}{r_{\mathrm{j}}}\right) n_{\mathrm{i}} n_{\mathrm{j}} \\
& -\alpha_{\mathrm{b}} \sum_{i=1}^{\infty} \frac{2 k_{\mathrm{b}} T}{3 \eta_{0}}\left(r_{\mathrm{i}}+r_{\mathrm{j}}\right)\left(\frac{1}{r_{\mathrm{i}}}+\frac{1}{r_{\mathrm{j}}}\right) n_{\mathrm{i}} n_{\mathrm{j}} \\
& +\frac{1}{2} \alpha_{\mathrm{s}} \sum_{i=1, I+j=k}^{i=j-1} \frac{4 \dot{\gamma}}{3}\left(r_{\mathrm{i}}+r_{\mathrm{j}}\right)^{3} n_{\mathrm{i}} n_{\mathrm{j}} \\
& -\alpha_{\mathrm{s}} \sum_{i=1, I+j=k}^{i=j} \frac{4 \dot{\gamma}}{3}\left(r_{\mathrm{i}}+r_{\mathrm{j}}\right)^{3} n_{\mathrm{i}} n_{\mathrm{j}} \\
& -\frac{3}{4} \frac{\pi \cdot \dot{\gamma}^{2}}{F_{0} N_{\mathrm{b}}} \frac{\eta \cdot d_{0}^{3}}{(k-1)}\left(\frac{k}{1-\varepsilon}-1\right) n_{\mathrm{k}}
\end{aligned}
$$

$$
\begin{aligned}
& +\frac{3}{2} \frac{\pi \cdot \dot{\gamma}^{2}}{F_{0} N_{\mathrm{b}}} \frac{\eta \cdot d_{0}^{3}}{(2 k-1)}\left(\frac{2 k}{1-\varepsilon}-1\right) n_{2 \mathrm{k}} \\
& +\frac{3}{4} \frac{\pi \cdot \dot{\gamma}^{2}}{F_{0} N_{\mathrm{b}}} \frac{\eta \cdot d_{0}^{3}}{(2 k-2)}\left(\frac{2 k-1}{1-\varepsilon}-1\right) n_{2 \mathrm{k}-1} \\
& +\frac{3}{4} \frac{\pi \cdot \dot{\gamma}^{2}}{F_{0} N_{\mathrm{b}}} \frac{\eta \cdot d_{0}^{3}}{2 k}\left(\frac{2 k+1}{1-\varepsilon}-1\right) n_{2 \mathrm{k}+1} .
\end{aligned}
$$

Here, $n_{\mathrm{k}}\left[\mathrm{m}^{-3}\right]$ and $r_{\mathrm{i}}[\mathrm{m}]$ are the concentration of clusters composed of $k$ particles and the cluster radius composed of $i$ particles. In order to predict the local cluster size distributions in a non-uniform flow, the convection of clusters and the local shear rate and the local viscosity causing the coagulation and break-up of the clusters are considered in the present model. The governing equations of the flow are two-dimensional continuity and momentum equations as follows

Continuity equation:

$$
\frac{\partial \rho U}{\partial x}+\frac{\partial \rho V}{\partial y}=0
$$

Momentum Equation:

$$
\begin{aligned}
& \frac{\partial \rho U}{\partial t}+\frac{\partial \rho U^{2}}{\partial x}+\frac{\partial \rho U V}{\partial y}=-\frac{\partial P}{\partial x}+\eta\left(\frac{\partial^{2} U}{\partial x^{2}}+\frac{\partial^{2} V}{\partial y^{2}}\right) \\
& \frac{\partial \rho V}{\partial t}+\frac{\partial \rho U V}{\partial x}+\frac{\partial \rho V^{2}}{\partial y}=-\frac{\partial P}{\partial y}+\eta\left(\frac{\partial^{2} U}{\partial x^{2}}+\frac{\partial^{2} V}{\partial y^{2}}\right)
\end{aligned}
$$

Here, $x$ and $y$ are streamwise and normal coordinates, respectively. $U, V[\mathrm{~m} / \mathrm{s}]$ are $x, y$-direction velocity components, respectively. $\rho\left[\mathrm{kg} / \mathrm{m}^{3}\right]$ is the density of suspensions. In this study, the density was assumed to be the same as that of solvent. The suspension viscosity was calculated by the particle cluster distributions as described later. The model predicting the cluster distributions with the consideration on convections is as follows

$$
\begin{aligned}
& \frac{\partial n_{\mathrm{k}}}{\partial t}+U \frac{\partial n_{\mathrm{k}}}{\partial x}+V \frac{\partial n_{\mathrm{k}}}{\partial y}= \\
& \frac{1}{2} \alpha_{\mathrm{b}} \sum_{i=1, i+j=k}^{i=k-1} \frac{2 k_{\mathrm{b}} T}{3 \eta_{0}}\left(r_{\mathrm{i}}+r_{\mathrm{j}}\right)\left(\frac{1}{r_{\mathrm{i}}}+\frac{1}{r_{\mathrm{j}}}\right) n_{\mathrm{i}} n_{\mathrm{j}} \\
& -\alpha_{\mathrm{b}} \sum_{i=1}^{\infty} \frac{2 k_{\mathrm{b}} T}{3 \eta_{0}}\left(r_{\mathrm{i}}+r_{\mathrm{k}}\right)\left(\frac{1}{r_{\mathrm{i}}}+\frac{1}{r_{\mathrm{k}}}\right) n_{\mathrm{i}} n_{\mathrm{k}} \\
& +\frac{1}{2} \alpha_{\mathrm{s}} \sum_{i=1, i+j=k}^{i=j-1} \frac{4 \dot{\gamma}}{3}\left(r_{\mathrm{i}}+r_{\mathrm{j}}\right)^{3} n_{\mathrm{i}} n_{\mathrm{j}} \\
& -\alpha_{\mathrm{s}} \sum_{i=1}^{\infty} \frac{4 \dot{\gamma}}{3}\left(r_{\mathrm{i}}+r_{\mathrm{k}}\right)^{3} n_{\mathrm{i}} n_{\mathrm{k}} \\
& -\frac{3}{4} \frac{\pi \cdot \dot{\gamma}^{2}}{F_{0} N_{\mathrm{b}}} \frac{\eta \cdot d_{0}^{3}}{(k-1)}\left(\frac{k}{1-\varepsilon}-1\right) n_{\mathrm{k}}
\end{aligned}
$$




$$
\begin{aligned}
& +\frac{3}{2} \frac{\pi \cdot \dot{\gamma}^{2}}{F_{0} N_{\mathrm{b}}} \frac{\eta \cdot d_{0}^{3}}{(2 k-1)}\left(\frac{2 k}{1-\varepsilon}-1\right) n_{2 \mathrm{k}} \\
& +\frac{3}{4} \frac{\pi \cdot \dot{\gamma}^{2}}{F_{0} N_{\mathrm{b}}} \frac{\eta \cdot d_{0}^{3}}{(2 k-2)}\left(\frac{2 k-1}{1-\varepsilon}-1\right) n_{2 \mathrm{k}-1} \\
& +\frac{3}{4} \frac{\pi \cdot \dot{\gamma}^{2}}{F_{0} N_{\mathrm{b}}} \frac{\eta \cdot d_{0}^{3}}{2 k}\left(\frac{2 k+1}{1-\varepsilon}-1\right) n_{2 \mathrm{k}+1} .
\end{aligned}
$$

In order to estimate the void fraction in a cluster and the cluster size, Usui suggested the following equation

$$
\varepsilon=\varepsilon_{\max }\left(1-k^{-0.4}\right)
$$

$\varepsilon_{\max }$ is the maximum void fraction estimated from the original solid fraction, $\phi_{0}[-]$, and the maximum solid fraction, $\phi_{\mathrm{p}, \max }$ as follows

$$
\varepsilon_{\max }=1-\frac{\phi_{0}}{\phi_{\mathrm{p}, \max }} .
$$

In this study, Simha's model ${ }^{16)}$ was applied to calculate suspension viscosities as shown in the following

$$
\eta=\eta_{0}(1+2.5 \lambda(\Gamma) \phi) .
$$

$\Gamma[-]$ is the ratio of the radius of primary particle and the cell radius. $\lambda(\Gamma)$ is the function of $\Gamma$ as follows

$$
\lambda(\Gamma)=\frac{4\left(1-\Gamma^{7}\right)}{4\left(1+\Gamma^{10}\right)-25 \Gamma^{3}\left(1+\Gamma^{4}\right)+42 \Gamma^{5}} .
$$

$\Gamma$ is estimated from apparent volume by the equation as follows

$$
\Gamma^{3}=\frac{\phi}{f^{3}\left[1-\left(\phi^{\frac{1}{3}} / f\right)\right]^{3}} .
$$

$f^{3}$ is the supposed volume fraction of total cell. In the case of $\phi$ equal to the maximum packing fraction, $\phi_{\mathrm{p}, \max }[-], \Gamma$ becomes unity and $\phi_{\mathrm{p}, \max }=f^{3} / 8$. By using the following equation, the apparent volume fraction is calculated from the cluster size distribution as follows

$$
\phi=\sum_{k} \frac{\pi}{6} d_{\mathrm{k}}^{3} n_{\mathrm{k}}
$$

Here, the cluster diameter including $k$ particles can be estimated as follows

$$
d_{\mathrm{k}}=d_{0}\left(\frac{k}{1-\varepsilon}\right)^{\frac{1}{3}} .
$$

Then the local viscosity can be estimated with the local cluster size distribution. Thus, the momentum equations are solved with the local viscosities.
The velocity and the pressure fields are at first computed with the solvent viscosity. From the velocity fields, the local shear rate can be obtained, and then the local cluster distribution is calculated. Thus, the local viscosity is recomputed and applied to the momentum equation set.

Figure1 shows the computational domain used in this study. The present model was applied to a two-dimensional backward-step flow with the step height, $H$, of $200 \mu \mathrm{m}$, the channel width, $W$, of $400 \mu \mathrm{m}$ and the channel length, $L$, of $0.07 \mathrm{~m}$. The origin was set on the bottom of the step. The mean velocity in the narrow path was set at $380 \mu \mathrm{m} / \mathrm{s}$. The step height Reynolds number was $1.45 \times 10^{-4}$ at the present condition. The second-order accurate central differencing scheme was applied to diffusion terms. Quadratic Upstream Interpolation for Convective Kinematics (QUICK) ${ }^{17)}$ was applied to the convection terms as the third-order upward differencing scheme. Semi Implicit Method for Pressure Linked Equation (SIMPLE) ${ }^{18)}$ was used to solve the pressure field. An implicit method was applied to the time marching, though the present result became steady.

Totally, $52 \times 34$ grids were assigned in the computational domain. The temperature of the fluid was set at $293 \mathrm{~K}$. The solvent viscosity and density were set at $0.5 \mathrm{~Pa} \cdot \mathrm{s}$ and $1.25 \mathrm{~g} / \mathrm{cm}^{3}$ to correspond the experiments. The primary particle diameter was set at $3.5 \mu \mathrm{m}$ (density:1.06 $\mathrm{g} / \mathrm{cm}^{3}$ ), which was also used for experiments. At inlet boundary, the velocity profile and cluster distributions were set at the same as those obtained by the experiment. At the outlet boundary, the streawise gradients of velocities and cluster distributions were assumed to be zero locally. On the wall, the velocities were set at zero and the gradients of cluster distributions were zero.

The present method needs very low computational load compared with Distinct Element Method (DEM) ${ }^{19,20)}$, and the immersed body method often used for computations on the flow with particles. In the present case, the computation time was about 30 minutes by a workstation equipped with a 3.33 $\mathrm{GHz}$ Intel Xeon dual core processor.

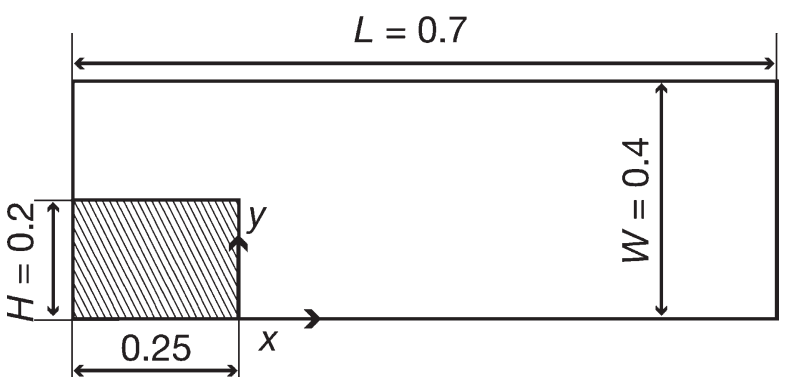

Fig. 1. Computational domain. 


\subsection{Experimental Methods}

In order to verify this model, a flow experiment was carried out using a micro channel. The micro channel, which has a rib at the mid-position of the channel, was produced. Polydimethylsiloxane (PDMS), a type of silicone elastomer, was used as a material of the channel. Figure 2 shows the geometry of the micro-channel. The length, the wide part width and the spanwise depth of the channel were $60,0.4$ and $0.1 \mathrm{~mm}$. The rib with the height of $0.02 \mathrm{~mm}$ and the length of $5 \mathrm{~mm}$ was mounted in the channel in order to make a non-uniform shear flow. The PDMS plate etched with that geometry was bonded to a borosilicate glass plate by a pressure bonding method. The experimental apparatus for the flow experiment is shown in Figure 3. The properly-prepared suspension was injected into the micro channel by a syringe pump keeping the flow rate and the flow was observed by an optical microscope (KH-1300, Hirox). The downstream part around rib was set as the test section for measurements of dispersion/aggregation and velocity characteristics. Polystyrene particles (diameter: $3.5 \mu \mathrm{m}$, density: $1.06 \mathrm{~g} / \mathrm{cm}^{3}$ ) were dispersed in $97 \mathrm{wt} \%$ glycerin solution. The solid volume fraction of particles, $\phi_{0}$, was set at 0.01 . The density of the glycerin solution was $1.25 \mathrm{~g} / \mathrm{cm}^{3}$. The viscosity of slurry was measured by a stress controlled rheometer (MCR-301, Anton-Paar). The measured viscosity was $0.5 \mathrm{~Pa} \cdot \mathrm{s}$ at $25^{\circ} \mathrm{C}$, which was the same as the glycerin solution because of the low solid fraction of 0.01 .

For analyze velocity distributions, Particle Image Velocimetry $(\mathrm{PIV})^{21,22)}$ was applied. In this study, the flow behaviors were observed and recorded by a high-speed camera (EX-F1,

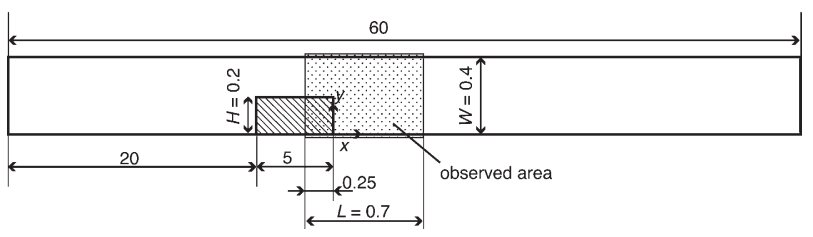

Fig. 2. Geometry of the micro-channel.

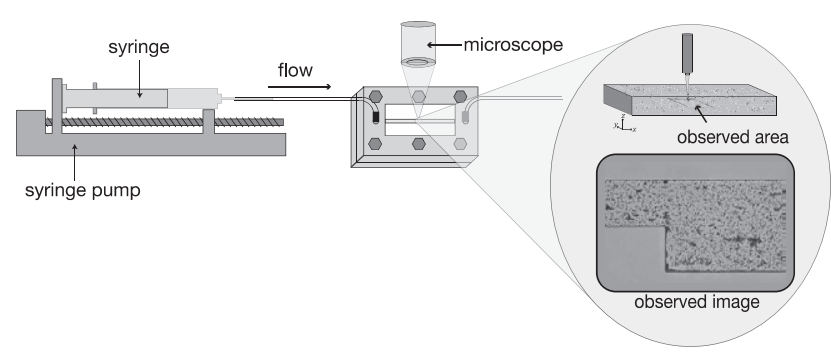

Fig. 3. Flow experiment apparatus using the micro-channel.
CASIO) with 600 fps and analyzed by FFT-based PIV method. The captured image sizes and interrogation area sizes were set at $512 \times 384$ and $16 \times 16$ pixels. In order to obtain velocity distribution, 200 images were used and averaged them.

\section{RESULTS AND DISCUSSION}

Figures 4 and 5 show the velocity vectors obtained by the numerical simulation and by the flow experiment, respectively.

In both cases of numerical and experimental results, the mean velocity at narrow section takes a value greater than one at broad section. In all cross-sectional distributions, the maximum velocity is observed at the center of the channel. These results indicate that the shear rate has local distribution. Figures 6 shows the cross-sectional velocity distributions at $x / H=-0.6,0.6$ and 1.2. In each figure, both of the numerical and the experimental results are plotted. In the upstream region of the step, the numerical velocity profile agrees well with that obtained by the experiment. After the step, the peak position of the velocity profile moves from $y / H=1.5$ to 1.4 at $x / H=0.6$. In the Figure 6 , the low velocity region was observed around $y / H=0.5$ at the position of $x / H=0.6$. In the

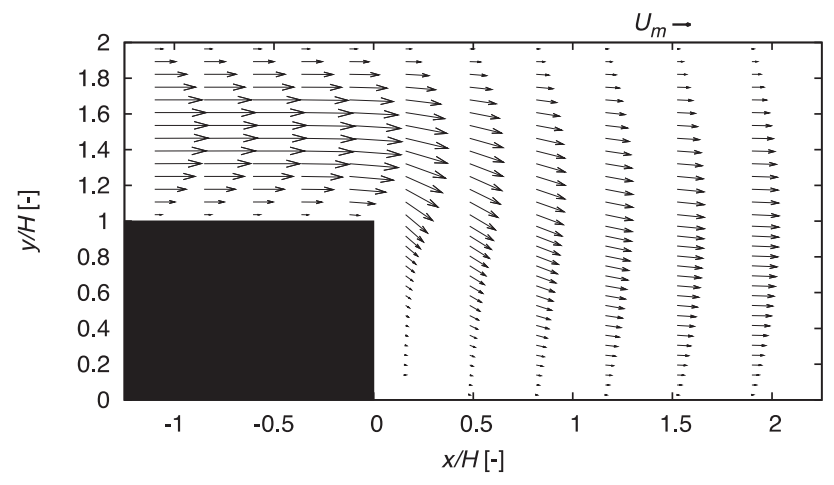

Fig. 4. Velocity vector distributions of numerical results.

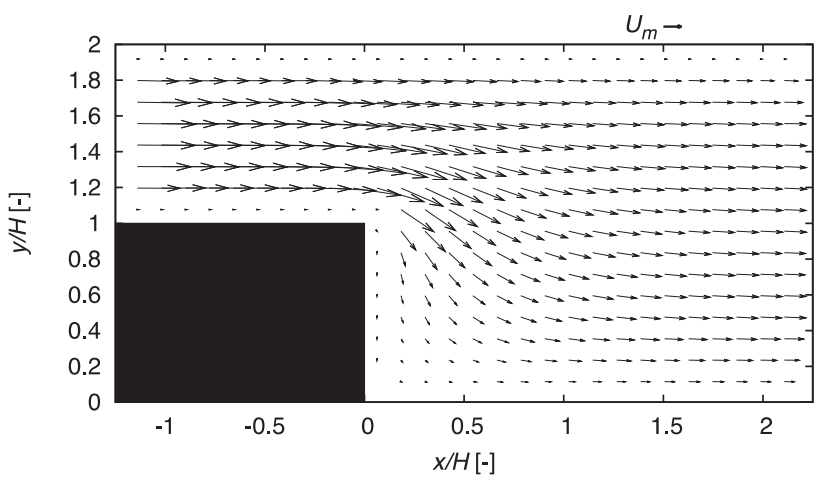

Fig. 5. Velocity vector distributions of experimental results. 
region, the experimental velocities take slightly higher than those of the numerical results. In the position of $x / H=1.2$, the peak moves to a lower position around $y / H=1.2$. The numerical velocities are estimated to be lower near wall region and to be higher around the peak position than those by the experiments. Those small differences are considered to be due to the three-dimensionality in experiments. The fluids and particles in the low velocity regions move spanwisely in experiments. This might cause the flow acceleration near wall region. On the contrary, the deceleration of the flow occurs in the core region. The present two-dimensional simulation could not express this effect. On the other hand, the reattachment point obtained by the present computation is at $x / H=0.51$. Biswas et $a l .{ }^{23)}$ reported the reattachment point locates at $x / H=0.42$ when the hydraulic diameter Reynolds number is lower than 1 in the same expansion ratio of the backwardfacing step of 0.5 . From this, the present numerical results on the velocity profiles can be found to be reasonable.

Figure 7 shows the mean cluster size distributions at $x / H=-0.6,0.6$ and 1.2 , respectively. Figure 8 shows the cumulative distributions at $y / H=0.2,0.5$ and 0.8 for the respective streamwise positions.
In the upstream region, the numerical results quite well agree with those obtained by the experiment both for the mean cluster size distribution and the cumulative distributions. At $x / H=0.6$, the numerical results show the high peak of the mean cluster size at $y / H=1.4$, where the shear rate becomes zero. On the other hand, the cluster size decreases near wall region, where the shear rate is high. When the shear rate is small, the particle aggregation occurs, and then the cluster size grows. On the contrary, the cluster breaks due to the high shear, and then, the cluster size tends to be small near wall region. The numerical results on the mean cluster size and on the cumulative size show the reasonable results. The experimental results in the downstream region of the step show slight difference with numerical ones. Especially, the difference becomes larger in the region near the lower wall where the spanwise flow might be intensified. This is caused by the decrease of the solid volume fraction due to threedimensional fluid motions. Figure 9 shows the local solid volume fraction distributions obtained numerically and experimentally. In the numerical results, the solid volume fraction is almost uniform in the normal direction, though the fraction becomes small near the lower wall. This low solid

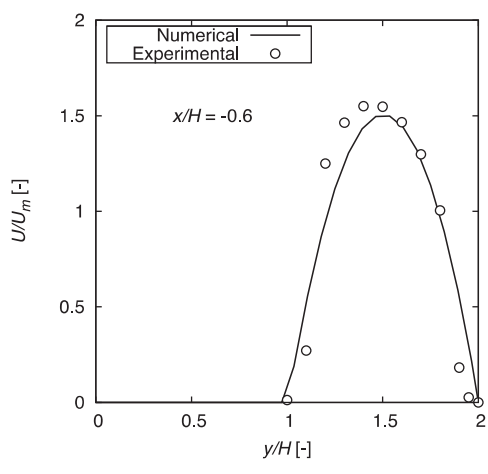

(a) $x / H=-0.6$

Fig. 6. Velocity profile.

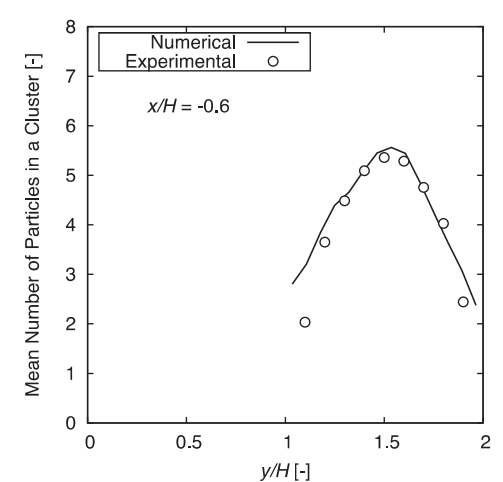

(a) $x / H=-0.6$

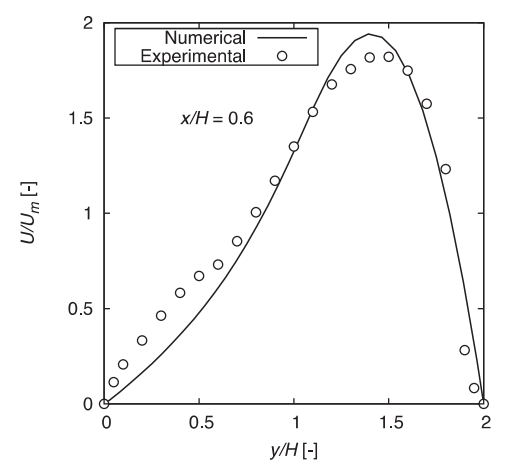

(b) $x / H=0.6$

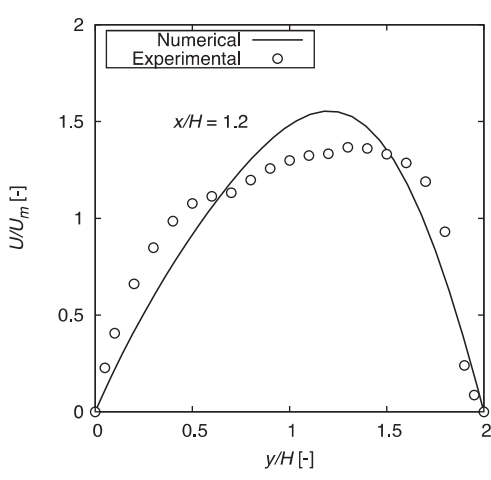

(c) $x / H=1.2$

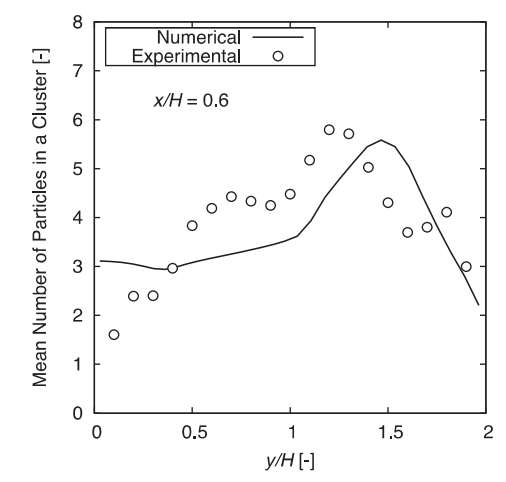

(b) $x / H=0.6$

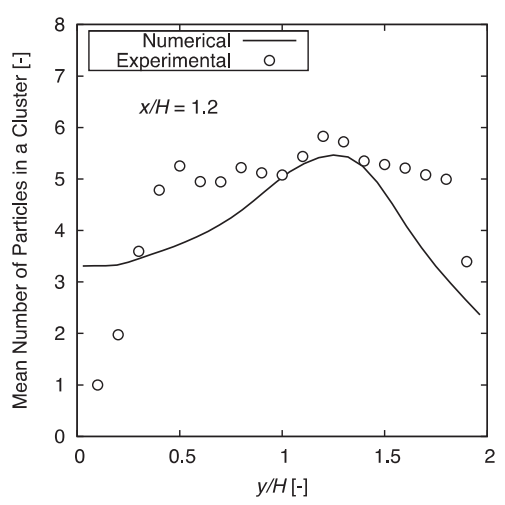

(c) $x / H=1.2$

Fig. 7. Mean number of particles in a cluster. 


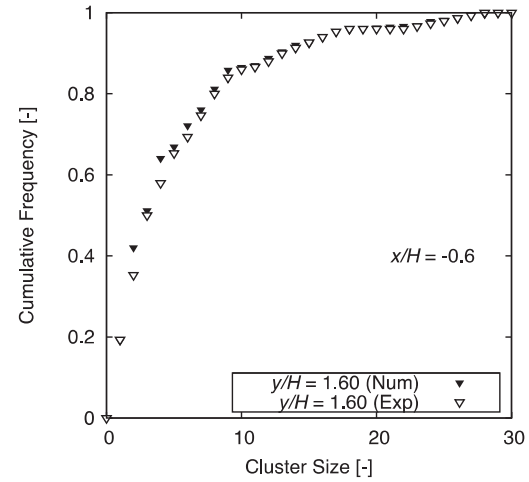

(a) $x / H=-0.6$

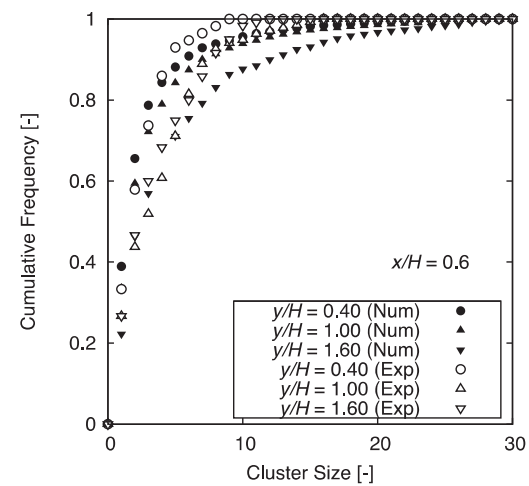

(b) $x / H=0.6$

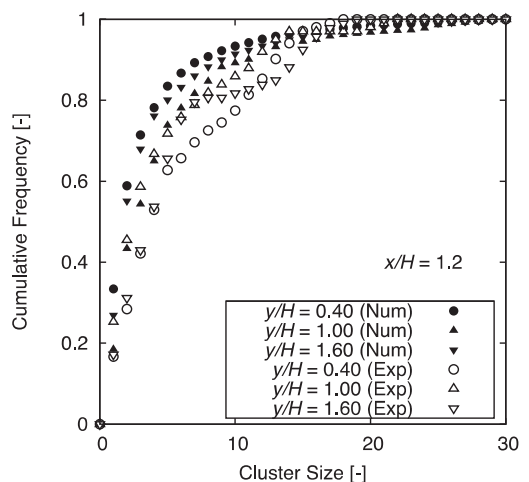

(c) $x / H=1.2$

Fig. 8. Cumulative frequency of the particle size distributions.

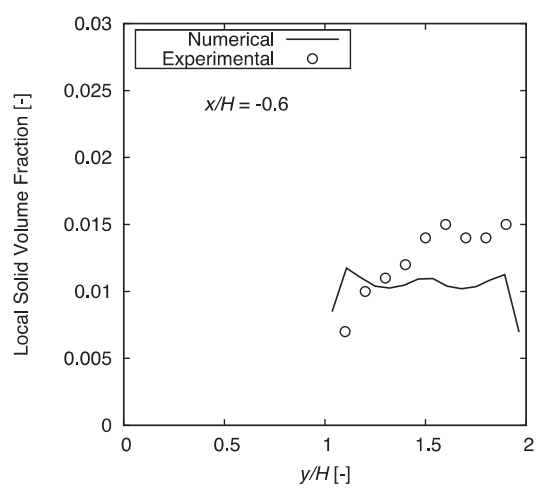

(a) $x / H=-0.6$

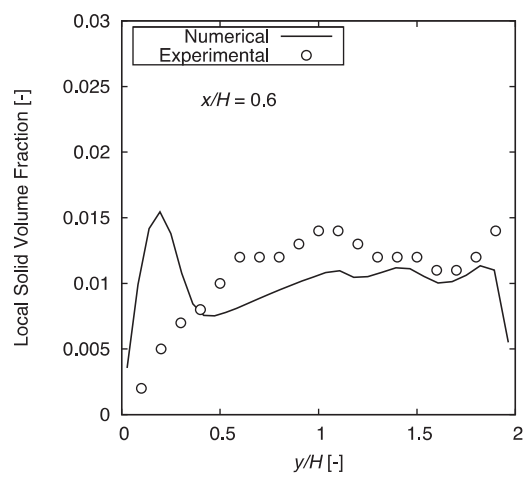

(b) $x / H=0.6$

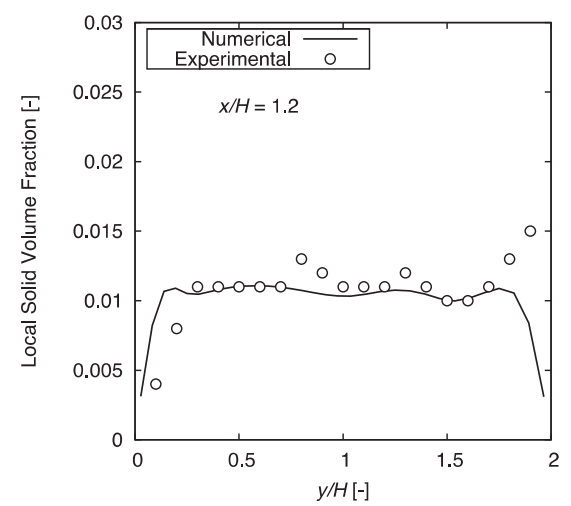

(c) $x / H=1.2$

Fig. 9. Local distribution of the solid volume fraction.

volume fraction causes to reduce the particle contacts. Thus, the cluster size in the lower normal positions is smaller than that of the numerical results. The decrease of volume fraction near the wall might relate also to the lift effect by the cluster rotation. The present computation does not take the lift force by the cluster rotation due to the shear into consideration. Thus, the concentration behavior of clusters to the middle region of the flow path is computed to be small in the present numerical works. This lift effect might also occur in the spanwise direction. The present computations are limited to be two-dimensional. This effect should be considered in the next study. However, the mean cluster size and the cumulative size distributions are comparably well estimated by the present study. The difference found in the cumulative size distribution is rather small at $x / H=0.6$, though the difference becomes slightly larger in the cumulative size distributions at $x / H=1.2$. As these differences are considered due to the three dimensionality of the flow, the present numerical simulation method can be a promising item for estimating the particle size distributions in a non-uniform flow.

\section{CONCLUSION}

In this paper, a model to predict to the local characteristics of particle dispersion/aggregation behaviors in a non-uniform shear flow was developed. The coupling calculation between coagulation behaviors and flow behaviors was performed. The flow experiment using a micro-channel was also performed for the verification of numerical simulations. From the comparison with numerical and experimental results, small disagreement was found in the downstream region around $x / H=1.2$. This can be considered to be due to the threedimensionality of the experimental flow. However, especially in the upstream region, the present numerical results well agree with the experimental data both in the mean cluster size and in the cluster size distributions. From this, the present numerical simulation method was concluded to be promising for estimating the particle dispersion/aggregation characteristics in a non-uniform shear flow. 


\section{NOTATIONS}

$d_{\mathrm{k}} \quad$ diameter of clusters include $k$ particles, $\mathrm{m}$

$d_{0} \quad$ diameter of primary particles, $\mathrm{m}$

$F_{0} \quad$ bonding energy, J

$f \quad$ characteristic distance of volume of particles at the maximum packing, -

$H \quad$ height of step, m

$k$ number of particles in a cluster, -

$k_{\mathrm{b}} \quad$ Boltzman number, $\mathrm{J} \cdot \mathrm{K}^{-1}$

$L \quad$ channel length, $\mathrm{m}$

$N_{\mathrm{b}} \quad$ number of bonds to be broken, -

$N_{0} \quad$ number of particles per unit, $\mathrm{m} / \mathrm{s}^{3}$

$n_{\mathrm{k}} \quad$ particle concentration include $k$ particles, $\mathrm{m} / \mathrm{s}^{3}$

Re Reynolds number, -

$r_{\mathrm{i}}, r_{\mathrm{j}} \quad$ radius of particle $i, j, \mathrm{~m}$

$T$ temperature, $\mathrm{K}$

$t$ time, $\mathrm{s}$

$U, V$ streamwise and normal velocity components, $\mathrm{m} / \mathrm{s}$

$U_{\mathrm{m}} \quad$ mean velocity, $\mathrm{m}$

$W \quad$ width of channel, $\mathrm{m}$

$x, y \quad$ streamwise and normal coordinates, $\mathrm{m}$

$\alpha_{\mathrm{b}}, \alpha_{\mathrm{s}}$ coefficients for Brownian and for shear coagulation, -

$\Gamma \quad$ ratio of particle radius and cell radius, -

$\dot{\gamma} \quad$ shear rate, $\mathrm{s}^{-1}$

$\varepsilon \quad$ void fraction, -

$\varepsilon_{\max }$ maximum void fraction, -

$\eta, \eta_{0} \quad$ slurry and solvent viscosities, $\mathrm{Pa} \cdot \mathrm{s}$

$\lambda$ coefficient on the volume fraction, -

$\rho$ density, $\mathrm{g} / \mathrm{cm}^{3}$

$\phi, \phi_{0} \quad$ apparent and actual solid volume fraction, -

$\phi_{\mathrm{p}, \max }$ the maximum packing fraction, -

\section{REFERENCES}

1) Smoluchowski M, Zeitschrift für Physikalische Chemie, 92 , 129, 168, (1917).
2) Swift DL, Friedlander S, J Colloid Sci, 19, 7, 621-647, (1964).

3) Higashitani $\mathrm{K}$, Tanaka $\mathrm{T}$, Yoshizo M, J Colloid and Interface Sci, 63, 3, 551-560, (1978).

4) Higashitani $\mathrm{K}$, Miyafusa $\mathrm{S}$, Matsuda $\mathrm{T}$, J Colloid and Interface Sci, 77, 1, 21-28, (1980).

5) Higashitani K, Ogawa R, Hosokawa G, Matsuno Y, J Chem Eng Jap, 15, 4, 299-304, (1982).

6) Parker DS, Kaufman WJ, Jenkins D, J Sanit Eng Div, 98, 1, 79-99, (1972).

7) Sonntag RC, Russel WB, J Colloid and Interface Sci, 113, 2, 399-413, (1986).

8) Sonntag RC, Russel WB, J Colloid and Interface Sci, 115, 2, 378-389, (1987).

9) Sonntag RC, Russel WB, J Colloid and Interface Sci, I, 2, 390-395, (1987).

10) Usui H, Kagaku Kogaku Ronbunshu, 25, 3, 459-465, (1999).

11) Usui H, Kinoshita S, Suzuki H, J Chem Eng Jap, 34, 3, 360$368,(2001)$.

12) Mustafa, Usui H, Shige I, Suzuki H, Kobayashi T, J Chem Eng Jap, 37, 1, 31-39, (2004).

13) Hasegawa E, Suzuki H, Kameyama K, Komoda Y, Usui H, Adv Powder Tech, 20, 2, 139-144, (2009).

14) Komoda Y, Kameyama K, Hasegawa E, Suzuki H, Usui H, Endo Y, Syudo A, Adv Powder Tech, 19, 6, 507-521, (2008).

15) Hasegawa E, Suzuki H, Komoda Y, Usui H, Nihon Reoroji Gakkaishi, 37, 4, 191-198, (2009).

16) Simha R, J Appl Phys, 23, 9, 1020-1024, (1952).

17) Leonard BP, Comput Meth Applied Mech Eng, 19, 59-98, (1979).

18) Patankar SV, "Numerical Heat Transfer and Fluid Flow", (1980), Hemisphere, Washington.

19) Cundall PA. A computer model for simulating progressive, large-scale movements in blocky rock systems. In Symposium Inteternational Society for Rock Mechics, Vol. 1, (1971).

20) Cundall PA, Geotechnique, 29, 1, 47-65, (1979).

21) Adrian R, Annu Rev of Fluid Mech, 23,1 , 261-304, (1991).

22) Willert CE, Gharib M, Exp Fluids, 10, 4, 181-193, (1991).

23) Biswas G, Breuer M, Durst F, J Fluids Eng, 126, 3, 362-374, (2004). 